\title{
ON A PROJECTIVE REPRESENTATION OF THE HALL-JANKO GROUP
}

\author{
BY J. H. LINDSEY, II
}

\author{
Communicated by Walter Feit, May 16, 1968
}

In the line of an investigation of the projective groups of degree six with complex coefficients, it was discovered that the Hall-Janko group, the unique simple group of order 604,800 , described in a paper by Marshall Hall and David Wales, has a projective representation of degree six. In fact, there exists a unique group, $G_{1}$, with center, $Z$, of order two, and $G_{1} / Z \cong G$, satisfying the following:

(A) the Sylow-7-subgroup of $G$ is normalized by an element of order four;

(B) the inverse image, under the homomorphism $G_{1} \rightarrow G_{1} / Z \cong G$, of a subgroup, $U_{3}(3)$, of $G$ of order 6048 is isomorphic to $Z \times U_{3}(3) . G_{1}$ has two conjugate, faithful, irreducible representations of degree six in the complex field. This representation can be written in $Q(\sqrt{ } 5, \sqrt{ }-7)$.

The character table of $G_{1}$ can be given, and it is unique. The degrees of the faithful, irreducible representations of $G_{1}$ are $6,6,64,64,50,50$, $216,14,84,126,126,252,56,56,448,350,336$.

Existence of $G_{1}$ was verified by taking $(\bmod 3)$ a representation of degree six. This modular representation restricted to $Z U_{3}(3)$ has a three dimensional invariant subspace, $V$. Then, under $G_{1}, V$ has one hundred images, which generators of $G_{1}$ permute exactly as their images permute the letters described in the Hall-Wales paper. This was checked by computer with a program written by George Shapiro.

Unique unitary matrices over the complex field were obtained for a six dimensional representation of generators of $G_{1}$ after the normalizer of a Sylow-7-subgroup was written in a normal form.

HARVARD UNIVERSITY 